\title{
L'interaction de la protéine Nef-VIH avec la protéine cellulaire $\beta$-COP: un détournement de la machinerie du trafic intracellulaire et d'exocytose au profit du VIH?
}

\begin{abstract}
Le virus de l'immunodéficience humaine (VIH), l'agent causal du SIDA, fait partie de la famille des lentivirus. Ces virus se distinguent des autres rétrovirus par le fait que, outre les gènes classiques $\mathrm{gag}$, pol, env, on trouve dans les lentivirus des gènes codant pour six protéines régulatrices, Tat, Rev, Nef, Vif, Vpr, Vpu. Tat et Rev sont des protéines dites auxiliaires qui sont indispensables, respectivement, pour l'activation transcriptionnelle du promoteur viral situé dans les LTR, et pour le transport des transcrits viraux non (génomique) ou partiellement épissés.
\end{abstract}

Nef, une protéine accessoire bien importante

Nef, Vif, Vpr, Vpu sont des protéines dites accessoires, car elles ne sont pas strictement indispensables pour la réplication du virus in vitro, dans des cellules en culture. Cependant, ces protéines semblent jouer in vivo un rôle important $[1,2]$. Cela a été particulièrement bien démontré pour Nef par l'équipe de Ronald Desrosiers (Cambridge, MA, USA), dans le modèle d'infection de macaques par le virus de l'immunodéficience simienne, SIV [3]. C'est ainsi qu'il est aujourd'hui établi que : (1) Nef est essentielle pour une bonne production virale in vivo. Des virus SIV défectueux pour Nef ne se répliquent pratiquement pas in vivo alors qu'ils se répliquent très bien in vitro. Par ailleurs, une infection par ces virus déficients en Nef protège contre une infection ultérieure par des virus sau- tion s'exerce in vivo en faveur de l'expression d'un gène nef fonctionnel. Si les singes sont infectés par un virus contenant un gène nef non fonctionnel du fait de la présence d'une mutation ponctuelle introduisant un codon stop prématuré, la réplication virale est aussi efficace que pour un virus sauvage ; mais les virus qui se répliquent ont, en fait, perdu le codon stop prématuré et sont revenus au type sauvage. Pour les virus ayant une délétion du gène nef, une telle réversion n'est pas possible, et ces virus ne se répliquent pas [3].

Les fonctions exercées par la protéine Nef au bénéfice du VIH ne sont pas encore élucidées à ce jour, mais un certain nombre d'observations importantes sont acquises, en particulier : (1) Nef diminue l'expression de CD4 à la surface des cellules infectées. Ce phénomène, en majeure partie lié à un accroissement de l'endocytose de $\mathrm{CD} 4$, est retrouvé quand Nef est exprimée isolément, en absence de tout contexte viral $[5,6]$. Cette diminution de l'expression en surface du récepteur du virus joue probablement un rôle majeur pour empêcher les réinfections multiples de cellules infectées par un virus, contribuant peut-être ainsi à l'optimisation ultérieure de la production de virus par ces cellules. (2) Nef augmente la production et l'infectivité des particules virales par des lymphocytes primaires périphériques in vitro. Cette influence positive de Nef sur la réplication virale pourrait s'expliquer par un effet sur l'assemblage, la maturation ou le transport et la libération des virions $[2,7]$.
Ces activités impliquent que Nef interagisse avec des facteurs cellulaires dans les cellules infectées. Identifier ces facteurs permettrait, outre la compréhension des mécanismes d'action de Nef, la mise en évidence de nouvelles cibles pour le développement de molécules antivirales.

Dans ce but, nous avons entrepris d'identifier les protéines cellulaires capables d'interagir avec Nef grâce à la mise en œuvre du système doublehybride dans la levure $S$. cerevisiae [8]. Parmi les ADNc ainsi isolés, l'un codait pour la protéine $\beta$-COP, indiquant que Nef interagit spécifiquement avec $\beta$-COP dans les cellules infectées [9]. Cette interaction détectée dans le système double hybride a été retrouvée in vitro par expression de Nef et de $\beta$-COP dans $E$. coli, et in vivo dans des cellules infectées : en effet, Nef et $\beta$-COP co-précipitent sous l'effet des anticorps anti-Nef. Ces observations démontrent que les deux protéines sont en étroite association dans les cellules infectées.

Nef- $\beta$-COP: le sens d'une interaction

Quels sont le sens et la fonction d'une telle association ? Le rôle physiologique de $\beta$-COP dans les cellules eucaryotes, d'une part [10], et les observations rapportées ci-dessus sur les effets de Nef, d'autre part, permettent dès maintenant de supposer que Nef pourrait interférer avec la machinerie de transport intracellulaire des protéines membranaires et d'exocytose. En effet, $\beta$-COP est un constituant majeur du manteau (coatomer) des COP-vésicules (vésicules sans cla- 
thrine), impliquées dans le transport vésiculaire entre le réticulum endoplasmique et le Golgi, entre les différents compartiments golgiens, entre le Golgi et la membrane plasmique $[11,12]$. Le rôle de $\beta$-COP est surtout bien documenté au niveau de l'exocytose mais l'implication éventuelle des coatomers dans certains processus d'endocytose indépendants de la clathrine n'est pas à exclure.

L'interaction de Nef avec $\beta$-COP pourrait donc expliquer, au moins partiellement, soit la perturbation du trafic intracellulaire de CD4 vers la membrane plasmique, d'une part, soit/et l'accroissement du transport intracellulaire et de la libération des virions, d'autre part. Si ces hypothèses se confirment, nous pourrions imaginer que le gène Nef, qui est conservé dans tous les lentivirus de primate, et dont l'ARN messager constitue de loin la majorité des ARN précoces du VIH [2], a été sélectionné au cours de l'évolution par ces virus, pour utiliser à leur profit la ma- chinerie de trafic intracellulaire et d'exocytose. Il est certain que si cette observation d'une interaction physique entre Nef et $\beta$-COP était confirmée également par des études fonctionnelles, les perspectives ouvertes seraient passionnantes. Nous devrions être fixés dans les prochains mois.

R.B.

S.B.

1. Subbramanian RA, Cohen E. Molecular biology of the human immunodeficiency virus accessory proteins. J Virol $1994 ; 68$ : 6831-5.

2. Cullen BR. The role of Nef in the replication cycle of the human immunodeficiency viruses. $\mathrm{Vi}$ rology $1994 ; 205: 1-6$.

3. Kestler HW, Ringler DJ, Mori K, Panicali DL, Sehgal PK, Daniel MD, Desrosiers RC. Importance of the nef gene for maintenance of high virus loads and for development of AIDS. Cell 1991 ; 65: 65l-62.

4. Daniel MD, Kirchoff F, Czajak SC, Sehgal PK, Desrosiers RC. Protective effect of a live attenuated SIV vaccine with a deletion in the nef gene. Science 1992 ; 258 : 1938-41.
5. Schwartz O, Dautry-Varsat A, Goud B, Maréchal V, Subtil A, Heard JM, Danos O. HIVl induces accumulation of CD4 in early endosomes. J Virol $1995 ; 69: 528-33$.

6. Aiken C Konner J, Landau NR, Lenburg ME, Trono D. Nef induces CD4 endocytosis: requirement for a critical dileucine motif in the membrane-proximal CD4 cytoplasmic domain. Cell $1994 ; 76$ : 853-64.

7. Miller MD, Warmerdam MT, Page KA, Fein berg MB, Green WC. Expression of the human immunodeficiency virus type 1 (HIV-1) nef gene during HIV-l production increases progeny particle infectivity independently of gpl60 or viral entry. J Virol 1995 ; 69 : 579- 84

8. Plessis A, Camonis JH. Le système double-hybride, mode d'emploi. médecine/sciences $1994 ; 10$ : I-IX.

9. Benichou S, Bomsel M, Bodéus M, Durand H, Doute M, Letourneur F, Camonis J, Benarous R. Physical interaction of the HIV-l Nef protein with $\beta$-COP, a component of non-clatrin coated vesicles essential for membrane traffic. J Biol Chem 1994 ; 269 : 30073-6.

10. Rothman JE. Mechanism of intracellular transport. Nature 1994 ; 372 : 55-63.

11. Gruenberg J. Mécanismes de la régulation du trafic membranaire. médecine/sciences 1993 ; 9 : 141-7.

12. Pauloin A. Bréfeldine A, protéines-G et transports membranaires golgiens. médecine/sciences $1993 ; 9: 917-25$.

\section{BRÈVES}

Vers un isolement de la cellule souche hématopoiétique? La cellule souche hématopoïétique est définie par son potentiel d'autorenouvellement et sa capacité de se différencier in vivo dans toutes les lignées hématopoïétiques. Son isolement, qui serait très utile à l'étude des processus de l'engagement des cellules en lignages, est en outre nécessaire pour améliorer les stratégies cliniques de greffe de moelle ou de thérapie génique [1$3]$; il est particulièrement difficile, car la caractérisation de ces cellules est fonctionnelle et non phénotypique, et toutes les méthodes utilisées n'ont jamais sélectionné qu'une population cellulaire qui reste hétérogène [4]. Un travail récent a abordé le problème d'une manière très élégante : puisque les cellules souches ne répondent pas aux stimulations prolifératives des cytokines, les cellules qui prolifè- rent sont des cellules déjà engagées dans un lignage; en combinant úne stimulation par les cytokines et un traitement antimétabolique, on pourrait provoquer la mort de ces cellules déjà engagées et, de ce fait, sélectionner celles qui résistent à l'action proliférative des cytokines [5]. Les cellules mononucléées de la moelle, triées ou non pour leur caractère CD34+, ont été traitées par le ligand Kit et l'interleukine 3, cytokines ayant des récepteurs sur les cellules primitives, en même temps que par le 5-fluoro-uracile pendant au moins sept jours. Une cellule sur $10^{5}$ cellules mononucléées de la moelle est ainsi sélectionnée. Ces cellules sélectionnées présentent les caractères phénotypiques des cellules souches: elles sont petites et denses; on n'y retrouve pas les antigènes spécifiques de cellules plus différenciées, seuls sont présents CD34 et c-Kit. Elles sont quiescentes et nécessitent du stroma médullaire pour leur culture, répondant à la définition des LTC-IC (long term, culture-initiating cell). Une cellule unique peut se différencier dans une culture à long terme en lignée lymphoïde et en lignée myéloïde. Il resterait encore à démontrer la capacité de repopulation dans une transplantation in vivo. La technique semble cependant efficace pour l'enrichissement/isolement de cellules aptes à cette repopulation.

[1. Coulombel L, Vainchenker W. médecine/sciences $1995 ; 11$ : 13-6.]

[2. Chabannon C, Mannoni P. médecine/sciences $1995 ; 11$ : 17-27.]

[3. Gluckman E, Carosella E. médecine/sciences $1995 ; 11$ : 28-34.]

[4. Hatzfeld J, et al. médecine/sciences $1993 ; 9$ : 1110-2.]

[5. Berardi AC, et al. Science 1995 ; 267: 104-8.] 